\title{
New Perspectives for Adjuvant Therapy in Severe Malaria
}

\author{
Mauro Prato ${ }^{1,2 *}$, Giuliana Giribaldi ${ }^{1}$ \\ ${ }^{1}$ Dipartimento di Genetica, Biologia e Biochimica, Università di Torino, Italy \\ ${ }^{2}$ Dipartimento di Neuroscienze, Università di Torino, Italy
}

Malaria is one of the most common parasitic diseases in the world, leading to over 1 million deaths/year. It mainly affects children under 5 years of age and pregnant women and can be fatal. The most virulent among the human malaria parasites is Plasmodium falciparum ( $P f)$, which is responsible for the bulk of the malaria-related morbidity and mortality. Symptoms of uncomplicated malaria include fever, headache and vomiting, whereas the major complications of severe malaria include Cerebral Malaria (CM), pulmonary edema, acute renal failure or severe anaemia. It is characterized by the binding of Infected Red Blood Cell (IRBC) to the vascular endothelium (cytoadherence) and to non-infected erythrocytes (rosetting). The accumulation of IRBC and non-infected RBC generates a reduction in the blood flow of the microvasculature, which results in tissue hypoxia and necrosis [1].

The current primary treatment for uncomplicated malaria involves the use of antimalarial drugs artemisinin, amodiaquine and quinine. Artemisinin is isolated from the plant Artemisia annua and represents, along with its derivatives, the most effective drug for the treatment of uncomplicated and severe malaria, with a rapid mechanism of action. Intravenous artesunate is at present the most effective treatment for $\mathrm{CM}$. Its superiority over quinine has been recently shown with the results of the SEAQUAMAT (South East Asian Quinine Artesunate Malaria Trial) and AQUAMAT study conducted in patients with CM in SE Asia and in Africa [2,3]. Artemisinin-Based Combination Therapy (ACT) is the main stay of treatment for uncomplicated malaria all over the world. However, there are confirmed reports of clinical resistance to artemisinin monotherapy and ACT from SE Asia; although the mechanism is not clear yet and the phenotypic characteristics of Pf strains resistant to artemisinin are unknown, these data envisage the tremendous risk of losing in the near future the best treatment for $\mathrm{CM}$ and the main component of ACT [4]. How such a risk could be avoided? What directions should be taken by current research? Are there any new targets which should be taken in account for urgent investigation and future clinical trials?

Every effort should be focused in trying to develop new fast acting antimalarials, but also to investigate new treatment regimens or adjunct therapies to manage the pathogenic manifestation of the disease once that the ability to reduce the parasite burden will be impaired by resistance or by the availability of less effective drugs. Unfortunately, to date there is no effective adjuvant therapy aimed at reducing symptoms of CM. In an effort to reduce malaria-related mortality, numerous adjunctive therapies that may alter malaria-induced abnormalities in physiology have been tested, as recently reviewed by John et al. [5]. These therapies may act directly on specific biologic pathways altered by malaria or more generally on end-stage factors produced in malaria by a number of different specific processes. Thus, several targets were chosen among factors believed to be key in the pathophysiological processes of severe malaria (e.g., elevated levels of TNFa or low levels of $\mathrm{NO}$ ) or agents that control end-stage factors associated with poor clinical outcomes (e.g., hypovolemia or metabolic acidosis). In a number of instances, these interventions were successful in reducing mortality in murine models of severe malaria, but results in human studies have been disappointing. Of all the interventions to date, only albumin infusion has led to a significant decrease in mortality and this finding was from a single small study.
The finding of new therapeutic targets is therefore extremely important. Recently, convincing evidence suggested that Matrix Metalloproteinases (MMPs) could be involved in the clinical outcome of uncomplicated towards severe malaria. These enzymes are produced by several cell types, including mononuclear and Endothelial Cells (EC) and play several functions through their proteolytic ability, including remodeling of sub-endothelial matrix, disruption of cerebral tight junctions and activation of several pro-inflammatory molecules [6]. As recently reviewed $[7,8]$, growing evidence on the involvement of MMPs in severe malaria became available from our group and others in the last years. Data from autopsies of CM patients showed that high levels of MMP-1 were present within the brain, whereas in a murine model of CM increased expression/activity of MMP-7, -2 and -9 was observed. In addition, we proposed a link between human MMP and malarial pigment haemozoin (HZ): HZ-fed human monocytes produced in vitro high levels of TNF $\alpha$, IL- $1 \beta$ and MIP- $1 \alpha$ that, in turn, induced MMP9 expression; the latter promoted the shedding into the extracellular environment of the soluble form of TNFa, thus creating a pathological loop involving inflammatory molecules and MMP. Also, it was recently shown that IRBC and HZ induced de-novo MMP-9 expression in human microvascular EC; and up-regulated the levels of MMP-1 and MMP-3 with a consequent increase in gelatinolytic activity. In all these systems, NF- $\kappa \mathrm{B}$ activation has been reported using both $\mathrm{HZ}$ or IRBC as stimuli.

Based on this evidence, new possible therapeutic targets for new adjuvant treatments for severe malaria are taking shape. The use of a wide-spectrum MMP-inhibitor (Batimastat, BB94) significantly increased the survival of mice with CM [9]. Additionally, high doses of dexamethasone significantly reduced inflammation in a murine model of malaria-associated lung pathology [10]. Finally, natural flavonoid quercetin, a kinase inhibitor with anti-malarial properties, was able to block HZ-induced up-regulation of MMP-9, TNFa and IL-1 $\beta$ [11]. Thus, in the next years the malaria research community should strongly take in account and expand the currently available knowledge on the role of these molecules in the etiopathogenesis of severe malaria and identify and test some new drugs able to inhibit these substrates, in a common effort aimed at finding new affordable adjuvant therapies for severe malaria.

\section{References}

1. Dondorp AM, Pongponratn E, White NJ (2004) Reduced microcirculatory flow

${ }^{*}$ Corresponding author: Prof. Mauro Prato, Dipartimento di Genetica, Biologia e Biochimica, Facoltà di Medicina e Chirurgia, Università degli studi di Torino, Via Santena 5 bis, 10126, Torino, Italy, Tel: +39-011-670-58-43; Fax: +39-011-670-5845; Email: mauro.prato@unito.it

Received April 02, 2012; Accepted April 03, 2012; Published April 09, 2012

Citation: Prato M, Giribaldi G (2012) New Perspectives for Adjuvant Therapy in Severe Malaria. J Bacteriol Parasitol 3:e105. doi:10.4172/2155-9597.1000e105

Copyright: (C) 2012 Prato $\mathrm{M}$, et al. This is an open-access article distributed under the terms of the Creative Commons Attribution License, which permits unrestricted use, distribution, and reproduction in any medium, provided the original author and source are credited. 
Citation: Prato M, Giribaldi G (2012) New Perspectives for Adjuvant Therapy in Severe Malaria. J Bacteriol Parasitol 3:e105. doi:10.4172/21559597.1000e105

in severe falciparum malaria: pathophysiology and electron-microscopic pathology. Acta Trop 89: 309-317.

2. Dondorp A, Nosten F, Stepniewska K, Day N, White N, et al. (2005) Artesunate versus quinine for treatment of severe falciparum malaria: a randomised trial. Lancet 366: 717-725.

3. Dondorp AM, Fanello Cl, Hendriksen IC, Gomes E, Seni A, et al. (2010) Artesunate versus quinine in the treatment of severe falciparum malaria in African children (AQUAMAT): an open-label, randomised trial. Lancet 376: 1647-1657.

4. Noedl H, Se Y, Schaecher K, Smith BL, Socheat D, et al. (2008) Evidence of artemisinin-resistant malaria in western Cambodia. N Engl J Med 359: 26192620.

5. John CC, Kutamba E, Mugarura K, Opoka RO (2010) Adjunctive therapy for cerebral malaria and other severe forms of Plasmodium falciparum malaria. Expert Rev Anti Infect Ther 8: 997-1008.

6. Cauwe B, Van den Steen PE, Opdenakker G (2007) The biochemical, biological, and pathological kaleidoscope of cell surface substrates processed by matrix metalloproteinases. Crit Rev Biochem Mol Biol 42: 113-185.
7. Prato M, Giribaldi G (2011) Matrix Metalloproteinase-9 and Haemozoin: Wedding Rings for Human Host and Plasmodium falciparum Parasite in Complicated Malaria. J Trop Med 2011: 628435.

8. Geurts N, Opdenakker G, Van den Steen PE (2012) Matrix metalloproteinases as therapeutic targets in protozoan parasitic infections. Pharmacol Ther 133: 257-279.

9. Van den Steen PE, Van Aelst I, Starckx S, Maskos K, Opdenakker G, et al. (2006) Matrix metalloproteinases, tissue inhibitors of MMPs and TACE in experimental cerebral malaria. Lab Invest 86: 873-888.

10. Van den Steen PE, Geurts N, Deroost K, Van Aelst I, Verhenne S, et al. (2010) Immunopathology and dexamethasone therapy in a new model for malariaassociated acute respiratory distress syndrome. Am J Respir Crit Care Med 181: 957-968.

11. Prato M, Gallo V, Giribaldi G, Aldieri E, Arese P (2010) Role of the NF-kB transcription pathway in the haemozoin- and 15-HETE-mediated activation of matrix metalloproteinase- 9 in human adherent monocytes. Cell Microbiol 12 1780-1791. 